\title{
Epidural Analgesia in the cytoreductive surgery combined with hyperthermic intraperitoneal chemotherapy - a risky practice?
}

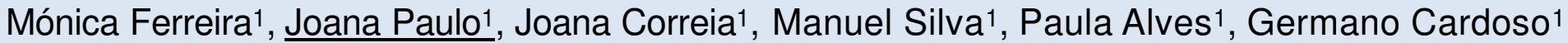

1 Instituto Português de Oncologia do Porto Francisco Gentil, EPE

\section{INTRODUCTION}

Cytoreductive surgery combined with heated intraperitoneal chemotherapy (HIPEC) has been improving prognosis of patients with peritoneal carcinomatosis.[1] This aggressive therapeutic approach has become an anesthetic challenge not only for hemodynamic stabilization during surgery but also for post-operative analgesia.[2] Since it is an extremely painful surgery, epidural analgesia presents itself as the recommended technique. However, HIPEC's influence on coagulation and hematological profile increases the risk of epidural hematoma or abscess which may limit epidural's analgesia role.[3]

Therefore, our aim is to evaluate safety and efficacy of epidural analgesia on HIPEC.

\section{MATERIAL AND METHODS}

This retrospective observational study was conducted from june 2016 to june 2017, through consulting of electronic clinical process and Acute Pain Unit database.

Patients under general anesthesia and epidural analgesia protocol (PCEA2) were included. This analgesic protocol included $1 \mathrm{~g}$ of paracetamol every 6 hours and a continuous perfusion through epidural catheter of ropivacaine $2 \mathrm{mg} / \mathrm{mL}$ and suphentanil 50 $\mathrm{ug} / \mathrm{mL}$ (rate of $5-15 \mathrm{~mL} / \mathrm{h}$, adjusted to patient and surgery). Patients bolus, between 3 to $5 \mathrm{~mL}$, had a 20 minutes' lockout period and the maximal dose was 60 $\mathrm{mL}$ through a period of 4 hours.

General anesthesia was induced with intravenous fentanil (2 to $5 \mathrm{ug} / \mathrm{kg}$ ), propofol (2 to $3 \mathrm{mg} / \mathrm{kg}$ ) and rocuronium $(0.6 \mathrm{mg} / \mathrm{kg})$. After induction, invasive blood pressure was monitored. Anesthesia was maintained with desflurane, rocuronium and ropivacaine $(2 \mathrm{mg} / \mathrm{mL})$ according to patient's needs. Quimioperfusion lasted 60 minutes. The neuromuscular blockage was reverted with sugammadex. During postoperative period, all patients were daily evaluated by Acute Pain Unit.

Pain was evaluated according to a qualitative scale: good analgesia if no or only mild pain were reported, reasonable analgesia if an episode of moderate pain and bad analgesia if severe or unbearable pain were reported during hospitalization.

\section{Results ANd Discussion}

\begin{tabular}{|cc}
47 & patients were included. \\
Demographic characteristics \\
Age & 57 years \\
& {$[28-79]$} \\
Female & $72 \%(n=34)$ \\
Male & $28 \%(n=13)$ \\
ASA 2 & $83 \%(n=39)$ \\
ASA 3 & $17 \%(n=8)$
\end{tabular}

\section{Post-operative period}

Duration of hospital 14 days

stay $\quad[7-40]$

$$
\text { Destination }
$$

Intermediate Care $\quad 77 \%$

Unit $\quad(n=36)$

Intensive Care Unit $\quad 23 \%$

$23 \%$
$(n=11)$

Complications during hospital stay

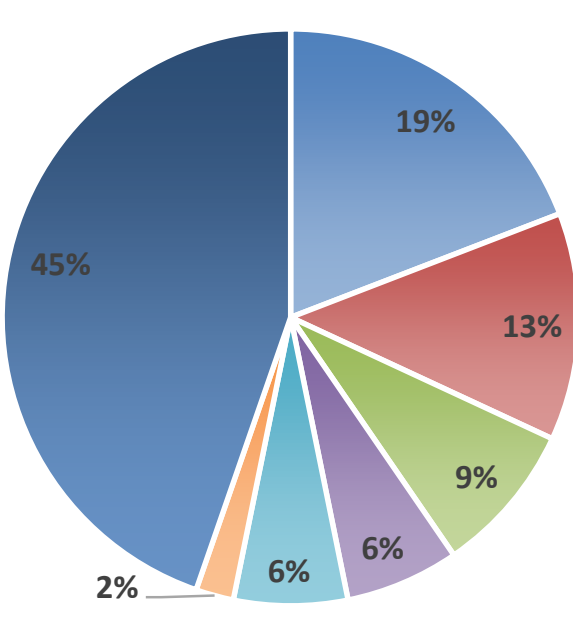

- PONV

- Hypotension

- Itching

- Paresthesia

- Accidental dislodgment

of cateter

Thrombocytopen

- No complications

\begin{tabular}{|ll|}
\hline \multicolumn{2}{|c}{ During surgery } \\
\hline Fentanyl dose & $0,20 \mathrm{mg}[0,10-0,40]$ \\
\hline Ropivacaine dose & $52 \mathrm{mg}[10-94]$ \\
\hline Use of vasopressors & $44,6 \%(\mathrm{n}=21)$ \\
\hline Blood loss & $300 \mathrm{~mL}[100$ a 1500$]$ \\
\hline Blood transfusion & $6,3 \%(\mathrm{n}=3)$ \\
\hline NV Prophylaxis & $93,4 \%(\mathrm{n}=44)$ \\
\hline Duration of surgery & $411^{\prime}(270-585)$ \\
\hline
\end{tabular}
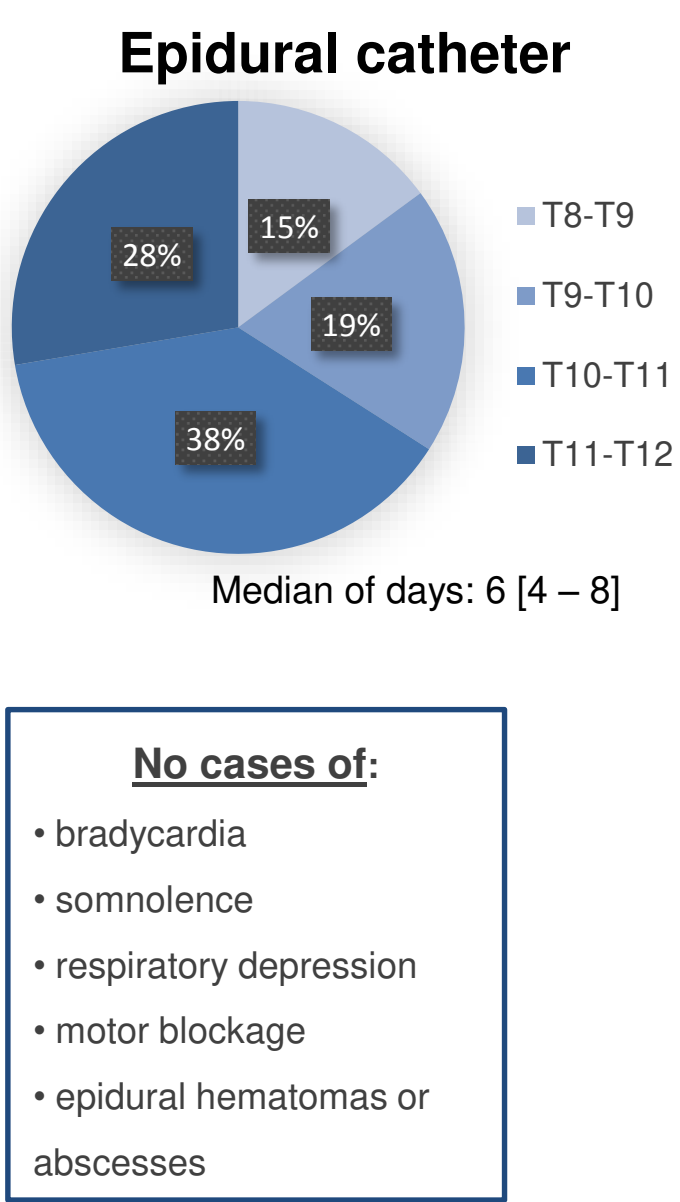

Analgesia quality

At rest

On movement

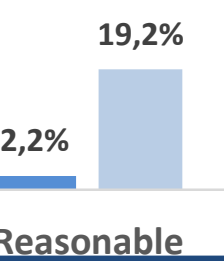

$0 \% \quad 0 \%$

\section{CONCLUSION}

With no major complications, our experience suggests that epidural analgesia is a safe, well-tolerated and effective technique for HIPEC surgery.

The higher incidence of nausea and vomiting and hypotension is related to the complexity of the surgery. Close monitoring is recommended in order to achieve satisfactory analgesia and to reduce the incidence of complications. 